\title{
An Entropic Order Quantity model with fuzzy holding cost and fuzzy disposal cost for perishable items under two component demand and discounted selling price
}

\author{
P.K. Tripathy \\ P.G. Dept. of Statistics, Utkal University \\ Bhubaneswar-751004, India \\ msccompsc@gmail.com \\ M. Pattnaik \\ Department of Business Administration, Utkal University \\ Bhubaneswar-751004, India \\ monalisha_1977@yahoo.com
}

\begin{abstract}
A new type of replenishment policy is suggested in an entropy order quantity model for a perishable product possessing fuzzy holding cost and fuzzy disposal cost. This model represents an appropriate combination of two component demand with discounted selling price, particularly over a finite time horizon. Its main aim lies in the need for an entropic cost of the cycle time is a key feature of specific perishable product like fruits, vegetables, food stuffs, fishes etc. To handle this multiplicity of objectives in a pragmatic approach, entropic ordering quantity model with discounted selling price during pre and post deterioration of perishable items to optimize its payoff is proposed. It has been imperative to demonstrate this model by analysis, which reveals some important characteristics of discounted structure. Furthermore, numerical experiments are conducted to evaluate the difference between the crisp and fuzzy cases in EOQ and EnOQ separately. This paper explores the economy of investing in economics of lot sizing in Fuzzy $E O Q$, Crisp EOQ and Crisp EnOQ models. The proposed paper reveals itself as a pragmatic alternative to other approaches based on two component demand function with very sound theoretical underpinnings but with few possibilities of actually being put into practice. The results indicate that this can become a good model and can be replicated by researchers in neighbourhood of its possible extensions.
\end{abstract}

Key Words: Stock dependent demand, Discounted selling price, Deterioration, Fuzzy.

\section{Introduction}

The predominant criterion in traditional inventory models is minimization of longrun average cost per unit time. The costs considered are usually fixed and variable ordering cost, holding cost, disposal cost. Costs associated with disorder in a system tied up in inventory are accounted for by including an entropy cost in the total costs. Entropy is frequently defined as the amount of disorder in a system. Jaber et al. (2008) proposed an analogy between the behaviour of production system and the behaviour of physical system.

In this paper we consider a continuous review, entropic order quantity model with fuzzy holding cost and fuzzy disposal cost. In traditional inventory models it has been common to apply fuzzy on demand rate, production rate and deterioration rate, whereas applying fuzzy in system cost usually ignored in Mahata et al. 
(2006) and Vujosevic (1996) et al. From practical experience, it has been found that uncertainty occurs not only due to lack of information but also as a result of ambiguity (vagueness) concerning the description of the sematic meaning of declaration of statements relating to an economic world. The fuzzy set theory was developed on the basis of non-random uncertainties. For this reason, we consider in our inventory model the holding cost and the disposal cost as the fuzzy member, since no researcher have discussed EnOQ model by introducing the holding cost and disposal cost as the fuzzy number.

Product perishability is an important aspect of inventory control. Deterioration in general, may be considered as the result of various effects on stock, some of which are damage, decay, decreasing usefulness and many more. Decaying products are of two types. Product which deteriorate from the very beginning and the products which start to deteriorate after a certain time. Lot of articles are available in inventory literature considering deterioration. Interested readers may consult the survey paper of Weatherford et al. (1992), Panda et al. (2009), Goyal et al. (2001) and Raafat (1991).

The constant demand model appears to have received considerably less attention in the inventory literature than the two component demand function model. In last two decades the variability of inventory level dependent demand rate on the analysis of inventory system was described by researchers like Pal et al. (1993) and Goswami et al. (1995). They described the demand rate as the power function of on hand inventory. There is a vast literature on stock development inventory and its outline can be found in the review article by Urban (2005) where he unified two types of inventory level dependent demand by considering a periodic review model. Researchers such as Chung et al. (2007), Goswami et al. (1995), Goyal et al. (2001), Pal et al. (1993), Raafat (1991) and Skouri et al. (2007) discussed the EOQ model assuming time value of money, demand rate, deterioration rate, shortages and so on a constant or probabilistic number or an exponential function.

In the development of an EOQ system, we usually omit the case of discounting on selling price. But in real world, it exists and is quite flexible in nature. On the other hand, in order to motivate customers to order more quantities, usually suppliers offer discount on selling prices both pre and post deterioration rate. Dave et al. (1995) developed an inventory model under continuous discount pricing. Khouja (2000) studied an inventory problem under the condition that multiple discounts can be used to sell excess inventory. Shah et al. (1993) mentioned that discount is considered temporarily for exponentially decaying inventory model. This paper represents the issue in details.

The purpose of this paper is to investigate the effect of the approximation made by using the average payoff when determining the optimal values of the policy variables. In this paper we focus exclusively on the cost of entropy with fuzzy holding cost and fuzzy disposal cost. A policy iteration algorithm is designed with the help of Deb (2000) and optimum solution is obtained through LINGO software. Numerical experiments are carried out to analyse the magnitude of the 
approximation error. We also consider the effect of fuzzy system in the total payoff. The remainder of this paper is organised as follows. In Section 2 describes the model formulation. Section 3 develops the fuzzy model. Section 4 provides mathematical analysis. In section 5, an illustrative numerical experiment is given to illustrate the procedure of solving the model. Finally in section 6 we make summary, concluding remarks and provide some suggestions for future research.

Table 1: Major Characteristics of Inventory Models on selected researches

\begin{tabular}{|l|c|c|c|c|c|c|}
\hline $\begin{array}{l}\text { Author(s) and } \\
\text { published } \\
\text { Year }\end{array}$ & $\begin{array}{c}\text { Structure } \\
\text { of the } \\
\text { Model }\end{array}$ & Deterioration & $\begin{array}{c}\text { Inventory } \\
\text { Model } \\
\text { Based on }\end{array}$ & $\begin{array}{c}\text { Discount } \\
\text { allowed }\end{array}$ & Demand & $\begin{array}{c}\text { Back- } \\
\text { logging } \\
\text { allowed }\end{array}$ \\
\hline $\begin{array}{l}\text { Mahata et al. } \\
(2006)\end{array}$ & Fuzzy & Yes (constant) & EOQ & No & Constant & No \\
\hline $\begin{array}{l}\text { Panda et al. } \\
(2009)\end{array}$ & Crisp & Yes (constant) & EOQ & Yes & $\begin{array}{c}\text { Stock } \\
\text { dependent }\end{array}$ & $\begin{array}{c}\text { Yes } \\
\text { (partial) }\end{array}$ \\
\hline $\begin{array}{l}\text { Jaber et al. } \\
\text { (2008) }\end{array}$ & Crisp & $\begin{array}{c}\text { Yes (on hand } \\
\text { inventory) }\end{array}$ & EnOQ & No & $\begin{array}{c}\text { Unit selling } \\
\text { price }\end{array}$ & No \\
\hline $\begin{array}{l}\text { Vujosevic et } \\
\text { al. (1996) }\end{array}$ & Fuzzy & No & EOQ & No & Constant & No \\
\hline $\begin{array}{l}\text { Chung et al. } \\
\text { (2007) }\end{array}$ & Crisp & Yes & EOQponential) & NOQ & $\begin{array}{c}\text { Selling } \\
\text { price }\end{array}$ & $\begin{array}{c}\text { Yes } \\
\text { (partial) }\end{array}$ \\
\hline $\begin{array}{l}\text { Skouri et al. } \\
\text { (2007) }\end{array}$ & Crisp & Yes (Weibull) & EOQ & No & Ramp & $\begin{array}{c}\text { Yes } \\
\text { (partial) }\end{array}$ \\
\hline $\begin{array}{l}\text { Present paper } \\
\text { (2010) }\end{array}$ & Fuzzy & $\begin{array}{c}\text { Yes } \\
\text { (Heaviside) }\end{array}$ & EnOQ & Yes & $\begin{array}{c}\text { Stock } \\
\text { dependent }\end{array}$ & No \\
\hline
\end{tabular}

\section{Mathematical Model with pre- and post deterioration discount on unit selling price}

At the beginning of the replenishment cycle the inventory level raises to $Q_{1}$. As time progresses it decreases due to instantaneous stock dependent demand up to the time $\tau$. After $\tau$ deterioration starts and the inventory level decreases for deterioration and constant demand. Ultimately inventory reaches zero level at $T_{1}$. Demand depends on the on hand inventory up to time $\tau$ from time of fresh replenishment beyond which it is constant and defined as follows. $R(I(t))=\left\{\begin{array}{l}a+b I(t), \quad t<\tau \\ a, t \geq \tau\end{array}\right.$ where $a>0$ is the initial demand rate independent of stock level and condition of inventory. $b>0$ is the stock sensitive demand parameter $I(t)$ is the instantaneous inventory level at time $t$. We assume that before the start of deterioration from time $t_{1}, r_{1},\left(0 \leq r_{1} \leq 1\right)$ is the percentage pre-deterioration discount offer on unit selling price of the product is given in order to boost the demand of fresh items. $\alpha_{1}=\left(1-r_{1}\right)^{-n_{1}}, n_{1} \in R$ is the effect of pre-deterioration discount on demand. Clearly, this discount is continued for the period of time $\left(\tau-t_{1}\right)$. As deterioration starts from $\tau, r_{2},\left(0 \leq r_{2} \leq 1\right)$ is the percentage discount offer on unit selling price during deterioration is provided to 
enhance the demand of decreased quality items. $\alpha_{2}=\left(1-r_{2}\right)^{-n_{2}}\left(n_{2} \in R\right.$ the set of real numbers), is the effect of discounted selling price on demand during deterioration. $\alpha_{2}$ is determined from priori knowledge of the seller such that the demand rate is influenced with the reduction rate of selling price. This discount is continued for the rest of the replenishment cycle. Then the behaviour of inventory level is governed by the following system of linear differential equations.

$$
\begin{aligned}
d I(t) / d t & =-a-b I(t) & & 0 \leq t \leq t_{1} \\
& =-\alpha_{1}(a+b I(t)) & & t_{1} \leq t \leq \tau \\
& =-\left[\alpha_{2} a+\theta I(t)\right] & & \tau \leq t \leq T_{1}
\end{aligned}
$$

The deterioration rate $\tilde{\theta}=\theta H(t-\tau),(0<\theta \leq 1)$ constant

Where $t$ is the time measured from the instant arrivals of a fresh replenishment indicating that the deterioration of the items begins after a time $\tau$ from the instant of the arrival in stock.

$H(t-\tau)$ is the well known Heaviside's function. $H(t-\tau)=\left\{\begin{array}{l}1, t \geq \tau \\ 0, \text { otherwise }\end{array}\right.$ With the initial boundary condition

$$
\begin{aligned}
& I(0)=Q_{1}, 0 \leq t \leq t_{1} \\
& I\left(T_{1}\right)=0, \tau \leq t \leq T_{1}
\end{aligned}
$$

Solving the equations,

$$
\begin{aligned}
I(t) & =\frac{a}{b}\left[e^{-b t}-1\right]+Q_{1}, e^{-b t}, 0 \leq t \leq t_{1} \\
& =\frac{a}{b}\left[e^{-\alpha_{1} b\left(t-t_{1}\right)-b t 1}-1\right]+Q_{1} e^{\alpha_{1} b\left(t_{1}-t\right)-b t_{1}}, t_{1} \leq t \leq \tau \\
& =\frac{a \alpha_{2}}{\theta}\left[e^{\theta\left(T_{1}-t\right)}-1\right], \tau \leq t \leq T_{1}
\end{aligned}
$$

Now, at the point $t=\tau$ we have from equation (5) and (6)

$$
Q_{1}=\left[\frac{a \alpha_{2}}{\theta}\left(e^{\theta\left(T_{1}-\tau\right)}-1\right)+\frac{a}{b}\right] \times e^{\alpha_{1} b\left(\tau-t_{1}\right)+b t_{1}}-\frac{a}{b}
$$

Holding cost and disposal cost of inventories in the cycle is,

$$
H C+D C=h \int_{o}^{t_{1}} I(t) d t+h \int_{t_{1}}^{\tau} I(t) d t+(h+\theta d) \int_{\tau}^{T_{1}} I(t) d t
$$

where $h$ is holding cost per unit per unit time and $d$ is disposal cost per unit.

Purchase cost in the cycle is given by $P C=c Q_{1}$.

where $c$ is per unit purchase cost of the product and $s$ is constant selling price of the product per unit $(s>c)$. 
Setup cost in the cycle is given by $\mathrm{OC}=C_{0}$.

Entropy generation rate must satisfy $S=\frac{d \sigma(t)}{d t}$ where, $\sigma(t)$ is the total entropy generated by time $t$ and $S$ is the rate at which entropy is generated. The entropy cost is computed by dividing the total commodity flow in a cycle of duration $T_{j}$.

The total entropy generated over time $T_{i}$ is $\sigma\left(T_{i}\right)=\int_{0}^{T_{i}} S d t, S=\frac{R(I(t))}{s}$

where $s$ is constant selling price of the product per unit $(s>c)$.

Entropy cost per cycle is

$$
E C\left(T_{i}\right)=\frac{D(\tau)}{\sigma(\tau)}+\frac{Q_{i} \text { With deterioration }}{\sigma\left(T_{i}\right)}(i=1,2,3)
$$

where $D(\tau)=\int_{0}^{t_{1}} R(I(t)) d t+\int_{t_{1}}^{\tau} R(I(t)) d t$

$$
\begin{aligned}
& Q_{i} \text { with deterioration }=Q_{1} \\
& \sigma(\tau)=\int_{0}^{\tau} S d t=\int_{0}^{t_{1}} \frac{R(I(t))}{s} d t+\int_{t_{1}}^{\tau} \frac{R(I(t))}{s} d t=\frac{1}{s} D(\tau) \\
& \sigma\left(T_{1}\right)=\int_{\tau}^{T_{1}} \frac{R(I(t))}{s} d t=\int_{\tau}^{T_{1}} \frac{a}{s} d t=\frac{a}{s}\left(T_{1}-\tau\right) \\
& E C=s+\frac{s Q_{1}}{a\left(T_{1}-\tau\right)}
\end{aligned}
$$

Total sales revenue in the order cycle can be found as

$$
S R=s\left[\int_{0}^{t_{1}}(a+b I(t)) d t+\alpha_{1}\left(1-r_{1}\right) \int_{t_{1}}^{\tau}[a+b I(t)] d t+\alpha_{2}\left(1-r_{2}\right) \int_{\tau}^{T_{1}} a d t\right]
$$

Thus total profit per unit time of the system is $\pi_{1}\left(r_{1}, r_{2}, t_{1}, T_{1}\right)$

$$
=\frac{1}{T_{1}}[S R-P C-H C-D C-E C-O C]
$$

On integration and simplification of the relevant costs, the total profit per unit time becomes

$$
\pi_{1}=F_{1}+F_{2} h+F_{3} d
$$

where

$$
F_{1}=\frac{1}{T_{1}}\left[\begin{array}{l}
s a t_{1}+s a \alpha_{2}\left(1-r_{2}\right)\left(T_{1}-\tau\right)+s\left(\left(Q_{1}+\frac{a}{b}\right)\left(1-e^{-b t_{1}}\right)-a t_{1}\right)+ \\
s\left(1-r_{1}\right)\left(Q_{1}+\frac{a}{b}\right) e^{-b t_{1}}\left(1-e^{\alpha_{1} b\left(t_{1}-\tau\right)}\right)-s-\frac{s Q_{1}}{a\left(T_{1}-\tau\right)}-c Q_{1}-C_{0}
\end{array}\right]
$$




$$
\begin{aligned}
& F_{2}=-\frac{1}{T_{1}}\left[\begin{array}{l}
\left(Q_{1}+\frac{a}{b}\right) \frac{1-e^{-b t_{1}}}{b}-\frac{a t_{1}}{b}+\frac{a}{b}\left(t_{1}-\tau\right)+\left(Q_{1}+\frac{a}{b}\right) e^{-b t}\left(\frac{1-e^{\alpha_{1} b\left(t_{1}-\tau\right)}}{b \alpha_{1}}\right) \\
+\frac{a \alpha_{2}}{\theta}\left(\frac{e^{\theta\left(T_{1}-\tau\right)}-1}{\theta}-\left(T_{1}-\tau\right)\right)
\end{array}\right] \\
& F_{3}=\frac{-a \alpha_{2}}{T_{1}}\left[\frac{e^{\theta\left(T_{1}-\tau\right)}-1}{\theta}-\left(T_{1}-\tau\right)\right]
\end{aligned}
$$

The pre-deterioration discount on selling price is to be given in such a way that the discounted selling price is not less that the unit cost of the product, i.e. $s\left(1-r_{1}\right)-c>0$. Similarly, $s\left(1-r_{2}\right)-c>0$.

Applying these constraints on unit total profit function we have the following maximization problem.

$$
\begin{aligned}
& \text { Maximize } \pi_{1}\left(r_{1}, r_{2}, t_{1}, T_{1}\right) . \\
& \text { Subject to }\left\{r_{1}, r_{2}\right\}<1-\frac{c}{s} \quad \forall r_{1}, r_{2}, t_{1}, T_{1}, \geq 0
\end{aligned}
$$

\section{Fuzzy model}

We replace the holding cost and disposal cost by fuzzy numbers $\tilde{h}$ and $\tilde{d}$ respectively. By expressing $\tilde{h}$ and $\tilde{d}$ as the normal triangular fuzzy numbers $\left(h_{1}, h_{0}, h_{2}\right)$ and $\left(d_{1}, d o, d_{2}\right)$, where, $h_{1}=h-\Delta_{1}$, ho $=h$, $h_{2}=h+\Delta_{2}, d_{1}=d-\Delta_{3}, d_{0}=d, d_{2}=d+\Delta_{4}$ such that $0<\Delta_{1}<h, 0<\Delta_{2}, 0<\Delta_{3}<d$, $0<\Delta_{4}, \Delta_{1}, \Delta_{2}, \Delta_{3}$ and $\Delta_{4}$ are determined by the decision maker based on the uncertainty of the problem.

The membership function of fuzzy holding cost and fuzzy disposal cost are considered as:

$$
\begin{gathered}
\mu_{\tilde{h}}(h)=\left\{\begin{array}{l}
\frac{h-h_{1}}{h_{0}-h_{1}}, h_{1} \leq h \leq h_{0} \\
\frac{h_{2}-h}{h_{2}-h_{0}}, h_{0} \leq h \leq h_{2} \\
0, \text { otherwise }
\end{array}\right. \\
\mu_{\tilde{d}}(d)=\left\{\begin{array}{l}
\frac{d-d_{1}}{d_{0}-d_{1}}, d_{1} \leq d \leq d_{0} \\
\frac{d_{2}-d}{d_{2}-d_{0}}, d_{0} \leq d \leq d_{2} \\
0 \quad, \text { otherwise }
\end{array}\right.
\end{gathered}
$$


Then the centroid for $\tilde{h}$ and $\tilde{d}$ are given by

$$
M_{\tilde{h}}=\frac{h_{1}+h_{o}+h_{2}}{3}=h+\frac{\Delta_{2}-\Delta_{1}}{3} \text { and } M_{\tilde{d}}=\frac{d_{1}+d_{o}+d_{2}}{3}=d+\frac{\Delta_{4}-\Delta_{3}}{3} \text { respectively. }
$$

For fixed values of $r_{1}, r_{2}, t_{1}$, and $T_{1}$, let

$$
Z(h, d)=F_{1}\left(r_{1}, r_{2}, t_{1} T_{1}\right)+F_{2}\left(r_{1}, r_{2}, t_{1}, T_{1}\right) h+F_{3}\left(r_{1}, r_{2}, t_{1}, T_{1}\right) d=y
$$

Let $h=\frac{y-F_{1}-F_{3} d}{F_{2}}, \frac{\Delta_{2}-\Delta_{1}}{3}=\psi_{1}$ and $\frac{\Delta_{4}-\Delta_{3}}{3}=\psi_{2}$

By extension principle the membership function of the fuzzy profit function is given by

$$
\begin{aligned}
\mu_{\tilde{z}(\tilde{h}, \tilde{d})}^{(y)} & =\operatorname{Sup}_{(h, d) \in Z^{-1}(y)}\left\{\mu_{\tilde{h}}(h) \vee \mu_{\tilde{d}}(d)\right\} \\
& =\operatorname{Sup}_{d_{1} \leq d \leq d_{2}}\left\{\mu_{\tilde{h}}\left(\frac{y-F_{1}-F_{3} d}{F_{2}}\right) \vee \mu_{\tilde{d}}(d)\right\}
\end{aligned}
$$

Now,

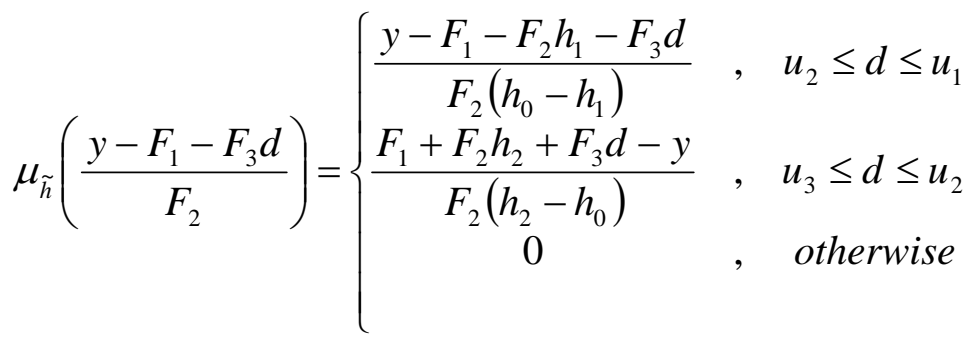

where,

$$
u_{1}=\frac{y-F_{1}-F_{2} h_{1}}{F_{3}}, u_{2}=\frac{y-F_{1}-F_{2} h_{0}}{F_{3}} \text { and } u_{3}=\frac{y-F_{1}-F_{2} h_{2}}{F_{3}}
$$

when $u_{2} \leq d$ and $d \leq u_{1}$ then $y \leq F_{1}+F_{2} h_{0}+F_{3} d_{0}$ and $y \geq F_{1}+F_{2} h_{1}+F_{3} d_{1}$. It is clear that for every $y \in\left[F_{1}+F_{2} h_{1}+F_{3} d_{1}, F_{1}+F_{2} h_{0}+F_{3} d_{0}\right], \mu_{y}(y)=P P^{\prime}$. From the equations (13) and (16) the value of $P P^{\prime}$ may be found by solving the following equation:

$$
\begin{gathered}
\frac{d-d_{1}}{d_{0}-d_{1}}=\frac{y-F_{1}-F_{2} h_{1}-F_{3} d}{F_{2}\left(h_{0}-h_{1}\right)} \\
\text { or } \quad d=\frac{\left(y-F_{1}-F_{2} h_{1}\right)\left(d_{0}-d_{1}\right)+F_{2} d_{1}\left(h_{0}-h_{1}\right)}{F_{2}\left(h_{0}-h_{1}\right)+F_{3}\left(d_{0}-d_{1}\right)}
\end{gathered}
$$

Therefore, $P P^{\prime}=\frac{d-d_{1}}{d_{0}-d_{1}}=\frac{y-F_{1}-F_{2} h_{1}-F_{3} d}{F_{2}\left(h_{0}-h_{1}\right)+F_{3}\left(d_{0}-d_{1}\right)}=\mu_{1}(y)$, (say).

When $u_{3} \leq d$ and $d \leq u_{2}$ then $y \leq F_{1}+F_{2} h_{2}+F_{3} d_{2}$ and $y \geq F_{1}+F_{2} h_{0}+F_{3} d_{0}$. It is evident that for every $y \in\left[F_{1}+F_{2} h_{0}+F_{3} d_{0}, F_{1}+F_{2} h_{2}+F_{3} d_{2}\right], \mu_{\tilde{y}}(y)=P P^{\prime \prime}$. From the 
equations (13) and (16), the value of $P P^{\prime \prime}$ may be found by solving the following equation:

$$
\frac{d_{2}-d}{d_{2}-d_{0}}=\frac{F_{1}+F_{2} h_{2}+F_{3} d-y}{F_{2}\left(h_{2}-h_{0}\right)}
$$

or, $\quad d=\frac{F_{2} d_{2}\left(h_{2}-h_{0}\right)-\left(F_{1}+F_{2} h_{2}-y\right)\left(d_{2}-d_{0}\right)}{F_{2}\left(h_{2}-h_{0}\right)+F_{3}\left(d_{2}-d_{0}\right)}$

Therefore, $P P^{\prime \prime}=\frac{d_{2}-d}{d_{2}-d_{0}}=\frac{F_{1}+F_{2} h_{2}+F_{3} d_{2}-y}{F_{2}\left(h_{2}-h_{0}\right)+F_{3}\left(d_{2}-d_{0}\right)}=\mu_{2}(y)$, (say).

Thus the membership function for fuzzy total profit is given by

$$
\mu_{\tilde{z}(\tilde{h}, \tilde{d})}(y)=\left\{\begin{array}{cc}
\mu_{1}(y) ; & F_{1}+F_{2} h_{1}+F_{3} d_{1} \leq y \leq F_{1}+F_{2} h_{0}+F_{3} d_{0} \\
\mu_{2}(y) ; & F_{1}+F_{2} h_{0}+F_{3} d_{0} \leq y \leq F_{1}+F_{2} h_{2}+F_{3} d_{2} \\
0 ; & \text { otherwise }
\end{array}\right.
$$

Now, let $P_{1}=\int_{-\infty}^{\infty} \mu_{\tilde{z}(\tilde{h}, \tilde{d})}(y) d y$ and $R_{1}=\int_{-\infty}^{\infty} y \mu_{\tilde{z}(\tilde{h}, \tilde{d})}(y) d y$

Hence, the centroid for fuzzy total profit is given by

$$
\begin{aligned}
& \tilde{\pi}_{1}=M_{\tilde{T P}}\left(r_{1}, r_{2}, t_{1}, T_{1}\right)=\frac{R_{1}}{P_{1}} \\
& =F_{1}\left(r_{1}, r_{2}, t_{1}, T_{1}\right)+F_{2}\left(r_{1}, r_{2}, t_{1}, T_{1}\right) h+F_{3}\left(r_{1}, r_{2}, t_{1}, T_{1}\right) d \\
& \quad+\psi_{1} F_{2}\left(r_{1}, r_{2}, t_{1}, T_{1}\right)+\psi_{2} F_{2}\left(r_{1}, r_{2}, t_{1}, T_{1}\right) \\
& M_{\tilde{T P}}\left(r_{1}, r_{2}, t_{1}, T_{1}\right)=F_{1}+\left(h+\psi_{1}\right) F_{2}+\left(d+\psi_{2}\right) F_{3}
\end{aligned}
$$

where, $F_{1}\left(r_{1}, r_{2}, t_{1}, T_{1}\right), F_{2}\left(r_{1}, r_{2}, t_{1}, T_{1}\right)$ and $F_{3}\left(r_{1}, r_{2}, t_{1}, T_{1}\right)$ are given by equations (9), (10) and (11).

Note that the two discounts $r_{1}$ and $r_{2}$ are given on constant unit selling price $s$ of the product. There may raise another case: the discount on unit selling price of the product from the start of deterioration may be given on the pre-deterioration discounted selling price $\left(1-r_{1}\right) s$.

The pre-deterioration discount on selling price is to be given in such a way that the discounted selling price is not less that the unit cost of the product, i.e. $s\left(1-r_{1}\right)-c>0$. Similarly, $s\left(1-r_{2}\right)-c>0$.

Applying these constraints on the unit total profit function in equation (21) we have the following maximization problem.

$$
\begin{aligned}
\text { Maximize } & M_{\tilde{T P_{1}}}\left(r_{1}, r_{2}, t_{1}, T_{1}\right) \\
\text { Subject to, } & \left\{r_{1}, r_{2}\right\}<1-\frac{c}{s} \\
& \forall \quad r_{1}, r_{2}, t_{1}, T_{1} \geq 0
\end{aligned}
$$


Our objective here is to determine the optimal values of $r_{1}, r_{2}, t_{1}$ and $T_{1}$ so as to maximize the unit profit function. It is very difficult to derive the results analytically. Thus some numerical methods must be applied to derive the optimal values of $r_{1}, r_{2}, t_{1}$ and $T_{1}$, hence the unit profit function. There are several methods to cope with constraint optimization problem numerically. But here we use penalty function method (Deb 2000) and LINGO software to derive the optimal values of the decision variables.

\section{Some Special Cases}

\section{Model with post deterioration discount on unit selling price}

We now consider the basic model by relaxing the assumption of discounted selling price from $t_{1}$ before deterioration. Discount on selling price will be given as soon as the deterioration starts.

In that case $t_{1}=\tau, r_{1}=0$ and $T_{1}=T_{2}$.

From equation (7) the initial inventory level is found as

$$
Q_{2}=\left[\frac{a \alpha_{2}}{\theta}\left(e^{\theta\left(T_{2}-\tau\right)}-1\right)+\frac{a}{b}\right] e^{b \tau}-\frac{a}{b}
$$

Substituting $t_{1}=\tau$ and $r_{1}=0$, we have the unit profit function of the system from equation (8) as

$$
\pi_{2}\left(r_{1}, T_{2}\right)=\frac{T P_{2}}{T_{2}}=F_{4}+F_{5} h+F_{6} d
$$

where,

$$
\begin{aligned}
& F_{4}=\frac{1}{T_{2}}\left[s a \tau+s\left(1-r_{2}\right) \alpha_{2} a\left(T_{2}-\tau\right)+s\left(\left(Q_{2}+\frac{a}{b}\right)\left(1-e^{-b \tau}\right)-a \tau\right)-s-\frac{s Q_{2}}{a\left(T_{2}-\tau\right)}-c Q_{2}-C_{0}\right] \\
& F_{5}=\frac{-1}{T_{2}}\left[-\frac{a \tau}{b}+\left(Q_{2}+\frac{a}{b}\right)\left(\frac{1-e^{-b \tau}}{b}\right)+\frac{a \alpha_{2}}{\theta}\left(\frac{e^{\theta\left(T_{2}-\tau\right)}-1}{\theta}-\left(T_{2}-\tau\right)\right)\right] \\
& F_{6}=\frac{-a \alpha_{2}}{T_{2}}\left[\frac{e^{\theta\left(T_{2}-\tau\right)}-1}{\theta}-\left(T_{2}-\tau\right)\right]
\end{aligned}
$$

There is post deterioration discount on selling price. Therefore, we have the fuzzy maximization problem.

$$
\begin{array}{ll}
\text { Maximize } & M_{\tilde{T P_{2}}}\left(r_{2}, T_{2}\right) \\
\text { Subject to } & r_{2}<1-\frac{c}{s} \\
& \forall \quad r_{2}, T_{2} \geq 0
\end{array}
$$

where, $M_{T P_{2}}\left(r_{2}, T_{2}\right)=F_{4}+\left(h+\psi_{1}\right) F_{5}+\left(d+\psi_{2}\right) F_{6}=\tilde{\pi}_{2}$. 


\section{Model for no discount on unit selling price}

In this case we consider that there is no pre-deterioration as well as no post deterioration discount on unit selling price. Substituting $t_{1}=\tau, T_{1}=T_{3}$ and $r_{1}=r_{2}=0$, we have from equation (7) the initial inventory level as,

$$
Q_{3}=\left[\frac{a}{\theta}\left(e^{\theta\left(T_{3}-\tau\right)}-1\right)+\frac{a}{b}\right] e^{b \tau}-\frac{a}{b}
$$

and from equation (8) total profit per unit time becomes,

$$
\pi_{3}\left(T_{3}\right)=\frac{T P_{3}}{T_{3}}=F_{7}+F_{8} h+F_{9} d
$$

where,

$$
\begin{aligned}
& F_{7}=\frac{1}{T_{3}}\left[s a \tau+s a\left(T_{3}-\tau\right)+s\left(\left(Q_{3}+\frac{a}{b}\right)\left(1-e^{-b \tau}\right)-a \tau\right)-s-\frac{s Q_{3}}{a\left(T_{3}-\tau\right)}-c Q_{3}-C_{0}\right] \\
& F_{8}=\frac{-1}{T_{3}}\left[-\frac{a \tau}{b}+\left(Q_{3}+\frac{a}{b}\right)\left(\frac{1-e^{-b \tau}}{b}\right)+\frac{a}{\theta}\left(\frac{e^{\theta\left(T_{3}-\tau\right)}-1}{\theta}-\left(T_{3}-\tau\right)\right)\right]
\end{aligned}
$$

and

$$
F_{9}=\frac{-a}{T_{3}}\left[\frac{e^{\theta\left(T_{3}-\tau\right)}-1}{\theta}-\left(T_{3}-\tau\right)\right]
$$

Since, no discount is provided on the unit selling price of the product, no constraints will be imposed on equation (31). The only constraint is the non-negative restriction for $T_{3}$. Hence,

$$
\text { Maximize } \begin{aligned}
M_{\check{T P_{3}}}\left(T_{3}\right) \\
\forall T_{3} \geq 0
\end{aligned}
$$

where, $M_{\tilde{T P_{3}}}\left(T_{3}\right)=F_{7}+\left(h+\psi_{1}\right) F_{8}+\left(d+\psi_{2}\right) F_{9}=\tilde{\pi}_{3}$.

\section{Model Analysis}

In the last session we have derived all possible unit profit functions arise from the associativity of deterioration and discount on unit selling price. In this section, we verify the applicability of the proposed discount structure. Let us consider the following theorem.

\section{Theorem - 1}

For $n_{1}=n_{2}=n, \tilde{\pi}_{1}>\tilde{\pi}_{2}$ if $r_{1}<\min \left\{1-\frac{c}{s}, \frac{\left(s-c-\frac{h+\psi_{1}}{b}\right) e^{b \tau}}{s}\right\}$. 


\section{Theorem - 2}

$$
\begin{aligned}
& \text { For } n_{1}=n_{2}=n, \tilde{\pi}_{2}>\tilde{\pi}_{3} \text { if } \\
& r_{2}<\min \left\{1-\frac{c}{s}, \frac{s(n-1)+n\left[\left(s b-\left(h+\psi_{1}\right)\right)\left(\frac{e^{b \tau}-1}{b}\right)-\frac{s}{a\left(T_{2}-\tau\right)}-c e^{b \tau}\right]}{s(n-1)}\right\} .
\end{aligned}
$$

A simple managerial indication is as follows: in pure inventory scenario if the product deteriorates after a certain time then it is always more profitable to apply both pre and post deterioration discount on unit selling price.

\section{Numerical Example}

The parameter values are $a=80, b=0.3, h=0.6, d=2.0, s=10.0, C_{0}=100.0, c=4.0$, $\theta=0.03, \tau=1.2, \mathrm{n}_{1}=\mathrm{n}_{2}=2.0, \quad{ }_{1}=0.1, \quad{ }_{2}=0.2, \quad{ }_{3}=0.5, \quad 4=0.8$ are listed in Table 2.

For the fuzzy entropic order quality model with both pre and post deterioration discounts, the pre-deterioration discount on unit selling price is $36.09 \%$ and discount starts at time 0.15239 and continued to $\tau=1.2$. Then the product starts to deteriorate. During this time in order to enhance inventory depletion rate, $47.88 \%$ discount is provided for remaining time of replenishment cycle. Profit per unit time is 665.2772 . The optimal order quantity and cycle length are 1373.823 and 2.7287 respectively. The unit profit and order quantity for only post deterioration discount are 565.3963 and 598.6194 less by 17.6656 and $129.4985 \%$, respectively. The cycle length is 2.7004 and $0.6764 \%$ less than that for model with both types of discounts. The post deterioration discount in this case $39.6475 \%$ and is less than $20.7618 \%$ from the model with both types of discounts whereas the model with no discount provides least payoff per unit time. The results are quite justified and agree with the model analysis at last section.

The relative differences in Table 3 is marked larger than the ones found in the previous experiments when the ordering policy is optimal. This indicates that there exists cases in which the relative difference is non-negligible. Based on the results in Table 2 and 3, we also note that the relative difference can be high when the ordering policy is optimal in the discounted selling price under pre and post deterioration model. While, with the same parameters, a positive ordering quantity is optimal in the post-deterioration discounted model and no discounted model. 
Table 2: Optimal values of the decision variables, order quantity and profit per unit time of different models

\begin{tabular}{|c|c|c|c|c|c|c|c|c|}
\hline $\begin{array}{l}\text { Nature of } \\
\text { Discount }\end{array}$ & $\begin{array}{l}\text { Decision } \\
\text { variables }\end{array}$ & FEnOQ & FEoQ & $\%$ change & CEnOQ & $\%$ change & CEOQ & $\%$ change \\
\hline \multirow{6}{*}{$\begin{array}{l}\text { With pre } \\
\text { and post } \\
\text { deterioration } \\
\text { discounts }\end{array}$} & $r_{1}$ & 0.3609 & 0.3810 & 5.5796 & 0.3700 & 2.5340 & 0.3898 & 8.0114 \\
\hline & $r_{2}$ & 0.4787 & 0.5502 & 14.9432 & 0.4950 & 3.3915 & - & - \\
\hline & $t_{1}$ & 0.1562 & 0.1482 & -5.1385 & 0.1774 & 13.5494 & 0.1709 & 9.4097 \\
\hline & $\mathrm{T}_{1}$ & 2.7287 & 2.3043 & -15.5536 & 2.7788 & 1.8358 & 1.2000 & -56.0233 \\
\hline & $Q_{1}$ & 1373.8230 & 1426.5010 & 3.8344 & 1501.2820 & 9.2776 & 376.4760 & -72.5964 \\
\hline & $\Pi_{1}$ & 665.2772 & 721.7426 & 8.4874 & 683.7764 & 2.7806 & 573.2467 & -13.8334 \\
\hline \multirow{6}{*}{$\begin{array}{l}\text { Post } \\
\text { deterioration } \\
\text { discount }\end{array}$} & $r_{1}$ & - & - & - & - & - & - & - \\
\hline & $r_{2}$ & 0.3964 & - & - & 0.4088 & 3.1246 & - & - \\
\hline & $t_{1}$ & - & - & - & - & - & - & - \\
\hline & $\mathrm{T}_{2}$ & 2.7004 & 1.2000 & -55.5636 & 2.7501 & 1.8420 & 1.2000 & -55.5630 \\
\hline & $Q_{2}$ & 598.6194 & 115.5402 & -80.6988 & 636.1641 & 6.2718 & 115.5402 & -80.6988 \\
\hline & $\Pi_{2}$ & 565.3963 & 459.9915 & -18.6426 & 575.8885 & 1.8557 & 461.8008 & -18.3226 \\
\hline \multirow{6}{*}{ No Discount } & $r_{1}$ & - & - & - & - & - & - & - \\
\hline & $r_{2}$ & - & - & - & - & - & - & - \\
\hline & $t_{1}$ & - & - & - & - & - & - & - \\
\hline & $\mathrm{T}_{3}$ & 2.9647 & 2.7129 & -8.4929 & 3.0344 & 2.3503 & 2.7822 & -6.1553 \\
\hline & $\mathrm{Q}_{3}$ & 323.3183 & 292.9956 & -9.3785 & 331.7506 & 2.6080 & 301.3188 & -6.8042 \\
\hline & $\Pi_{3}$ & 508.4216 & 520.2062 & 2.3178 & 513.1098 & 0.9221 & 524.4967 & 3.1617 \\
\hline
\end{tabular}

Table 3: Optimal values of Fuzzy EnOQ models $(i=1,2,3)$

\begin{tabular}{|l|c|c|r|r|r|}
\hline $\begin{array}{l}\text { Decision } \\
\text { Variable }\end{array}$ & $\begin{array}{c}\text { Both } \\
\text { Deterioration } \\
\text { Discount }\end{array}$ & $\begin{array}{c}\text { Post } \\
\text { Deterioration } \\
\text { Discount }\end{array}$ & $\begin{array}{c}\% \\
\text { Change }\end{array}$ & $\begin{array}{c}\text { No } \\
\text { Discount }\end{array}$ & \% Change \\
\hline $\mathrm{T}_{\mathrm{i}}$ & 2.728 & 2.7004 & -1.0359 & 2.9647 & 8.6492 \\
\hline $\mathrm{Q}_{\mathrm{i}}$ & 1373.823 & 598.6194 & -56.4267 & 323.3183 & -76.4657 \\
\hline$\Pi_{\mathrm{i}}$ & 665.277 & 565.3963 & -15.0134 & 508.4216 & -23.5774 \\
\hline
\end{tabular}

\section{Conclusion}

We have presented an entropic order quantity model with fuzzy holding cost and fuzzy disposal cost for perishable items under two component demand in which the criterion is to optimise the expected total discounted finite horizon payoff. To compute the optimal values of the policy parameters a simple and quite efficient policy model was designed. Theorem 1 and 2 effectively determine the optimal discount rate $r_{1}^{*}$ and $r_{2}^{*}$ for all the different cases. Finally, in numerical experiments the solution from the discounted model evaluated and compared to the solutions of other different traditional EOQ policies. 
However, we saw few performance differences among a set of different inventory policies in the existing literature. Although there are minor variations that do not appear significant in practical terms, at least when solving the single level, uncapacitated version of the lot sizing problem. From our analysis it is demonstrated that the retailer's profit is highly influenced by offering pre- and post discount on selling price. The results of this study give managerial insights to decision maker developing an optimal replenishment decision for deteriorating product. Compensation mechanism should also be included to induce collaboration between retailer and dealer in a meaningfull supply chain.

In general, for normal parameter values the relative payoff differences seem to be fairly small. The optimal solution of the suggested entropic order quantity model has a higher total payoff as compared with optimal solution for the traditional EOQ policy. Conventional wisdom suggests that workflow collaboration in a fuzzy entropic model in a varying deteriorating product in market place are promising mechanism and achieving a cost effective replenishment policy.

The approach proposed in the paper based on EnOQ model seems to be a pragmatic way to approximate the optimum payoff of the unknown group of parameters in inventory management problems. The assumptions underlying the approach are not strong and the information obtained seems worthwhile. Investigating optimal policies when demand are generated by other process and designing models that allow for several orders outstanding at a time, would also be challenging tasks for further developments. It's use may restrict the model's applicability in the real world. Future direction may be aimed at considering more general deterioration rate or demand rate. Uses of other demand side revenue boosting variables such as promotional efforts are potential areas of future research. The proposed paper reveals itself as a pragmatic alternative to other approaches based on two component demand function with very sound theoretical underpinnings but with few possibilities of actually being put into practice. The results indicate that this can become a good model and can be replicated by researchers in neighbourhood of its possible extensions. As regards future research, one other line of development would be to allow shortage and partial backlogging in the discounted model.

\section{References}

1. Chung YD, Tsu PH, Liang Y, (2007). Determining optimal selling price and lot size with a varying rate of deterioration and exponential partial backlogging. Eur. J. Oper. Res. 181: 668-678.

2. Dave DS, Fitzapatrick KE, Baker JR, (1995). An advertising inclusive production lot size model under continuous discount pricing comput. Ind. Eng. 30:147-159.

3. Deb K, (2000). Optimization for engineering design. Prentice-Hall of India. New Delhi. 
4. Goswami A, Choudhury KS, (1995). An EOQ model for deteriorating items with linear time dependent demand rate and shortages under inflation and time discounting J. Oper. Res. Soc. 46(6): 771.

5. Goyal SK, Giri BC (2001). Recent trends in modelling of deteriorating inventory. Eur. J. Oper. Res. (134):1-16.

6. Jaber MY, Bonney M, Rosen MA, Moualek I, (2008). Entropic order quantity (EnOQ) model for deteriorating items. Applied mathematical modelling.

7. Khouja M (2000). Optimal ordering, discounting and pricing in the single period problem. Int. J. Prod. Econ. 65:201-216.

8. Mahata GC, Goswami A, (2006). Production lot size model with fuzzy production rate and fuzzy demand rate for deteriorating item under permissible delay in payments. J. of Oper. Res. Soc. of India (43): 359-375.

9. Pal S, Goswami K, Chaudhuri KS, (1993). A deterministic inventory model for deteriorating items with stock dependent demand rate. J. Prod. Econ. 32:291-299.

10. Panda S, Saha S, Basu M, (2009). An EOQ model for perishable products with discounted selling price and stock dependent demand. CEJOR (17): 31-53.

11. Raafat E, (1991). Survey of Literature on continuously deteriorating inventory model J. Oper. Res. Soc. 42: 27-37.

12. Shah $\mathrm{NH}$, Shah $\mathrm{YK}$, (1993). An EOQ model for exponentially decaying inventory under temporary price discounts, cahiers du CERO 35: 227-232.

13. Skouri K., Konstantaras I., PapaChristos S., Ganas I., (2007). Inventory models with ramp type demand rate, partial backlogging and weibull deterioration rate. Euro. Jour. of Oper. Res.

14. Urban TL, (2005). Inventory model with inventory level dependent demand a comprehensive review and unifying theory, Eur. J. Oper. Res. 162: 792-804.

15. Vujosevic M, Petrovic D, Petrovic R, (1996). EOQ formula when inventory cost is fuzzy Int. Jour. of Prod. Econ. (45): 499-504.

16. Weatherford LR, Bodily SE, (1992). A taxonomy and research Overview of Perishable asset revenue management: yield management, overbooking, and pricing, Oper Res. 40:831-844. 


\section{Theorem - 1}

$$
\text { For } n_{1}=n_{2}=n, \tilde{\pi}_{1}>\tilde{\pi}_{2} \text { if } r_{1}<\min \left\{1-\frac{c}{s}, \frac{\left(s-c-\frac{h+\psi_{1}}{b}\right) e^{b \tau}}{s}\right\} \text {. }
$$

\section{Proof:}

The values of $\tilde{\pi}_{1}$ and $\tilde{\pi}_{2}$ for fixed $r_{1}$ and $r_{2}$ are always less than those for optimal $r_{1}$ and $r_{2}$. Thus it is sufficient to show that $\tilde{\pi}_{1}>\tilde{\pi}_{2}$ for fixed $r_{1}$ and $r_{2}$. Note that $T_{1}$ and $T_{2}$ are cycle lengths for the models with pre and post deterioration discounts and only post deterioration discount on unit selling price. Since the predeterioration discount on selling price is additional, demand of fresh items must be enhance and hence the inventory depletion rate must increase. Thus it is obvious that $T_{2}$ is always greater than $T_{1}$ from equations (21) and (29), we have

$$
\tilde{\pi}_{1}-\tilde{\pi}_{2}=\frac{\tilde{T P}}{T_{1}}-\frac{\tilde{T P}}{T_{2}} \geq \frac{\tilde{T P}-\tilde{T P}}{T_{2}}
$$

Therefore, it is sufficient to show that $\frac{\tilde{T P}_{1}-\tilde{T P}_{2}}{T_{2}}>0$, Now,

$$
\begin{aligned}
& \frac{\tilde{T P_{1}}-\tilde{T P}}{T_{2}}=\frac{1}{T_{2}}\left[-\left(a \alpha_{2}\left(T_{2}-\tau\right)+\frac{a}{b}\right)\left[\left(s-c-\left(\frac{h+\psi_{1}}{b}\right)\right) e^{b \tau}+s\left(1-r_{2}\right)-\frac{s b-\left(h+\psi_{1}\right)}{b}-\frac{s e^{b \tau}}{a\left(T_{2}-\tau\right)}\right]\right. \\
& +\left(a \alpha_{2}\left(T_{1}-\tau\right)+\frac{a}{b}\right)\left[\left(s-c-\frac{h+\psi_{1}}{b}\right) e^{\alpha_{1} b\left(\tau-t_{1}\right)+b t_{1}}+s\left(1-r_{2}\right)+\left[s\left(1-r_{1}\right) \alpha_{1} b-\left(h+\psi_{1}\right)\right]\right. \\
& \left.\left.\frac{e^{\alpha_{1} b\left(\tau-t_{1}\right)}-1}{b \alpha_{1}}-e^{\alpha_{1} b\left(\tau-t_{1}\right)}\left(\frac{s b-\left(h+\psi_{1}\right)}{b}\right)\right]-\frac{s e^{\alpha_{1} b\left(\tau-t_{1}\right)}}{a\left(T_{1}-\tau\right)}-\frac{a}{b}\left(T_{2}-\tau\right)\right] \\
& =\frac{1}{\left(s-c-\frac{h+\psi_{1}}{b}\right) e^{b \tau}+s\left(1-r_{2}\right)-\left(\frac{s b-\left(h+\psi_{1}\right)}{b}\right)-\frac{s e^{b \tau}}{a\left(T_{2}-\tau\right)}} \times \\
& {\left[\left(s-c-\frac{h+\psi_{1}}{b}\right) e^{\alpha_{1} b\left(\tau-t_{1}\right)+b t_{1}}+s\left(1-r_{2}\right)+\left[s\left(1-r_{1}\right) \alpha_{1} b-\left(h+\psi_{1}\right)\right] \frac{e^{\alpha_{1} b\left(\tau-t_{1}\right)}-1}{b \alpha_{1}}-e^{\alpha_{1} b\left(\tau-t_{1}\right)}\left(\frac{s b-\left(h+\psi_{1}\right)}{b}\right)-\frac{s e^{\alpha_{1} b\left(\tau-t_{1}\right)}}{a\left(T_{1}-\tau\right)}\right]} \\
& >\frac{a \alpha_{2}\left(T_{2}-\tau\right)+\frac{a}{b}}{a \alpha_{2}\left(T_{1}-\tau\right)+\frac{a}{b}}
\end{aligned}
$$

But $T_{2}>T_{1}$, therefore, $\frac{a \alpha_{2}\left(T_{2}-\tau\right)+\frac{a}{b}}{a \alpha_{2}\left(T_{1}-\tau\right)+\frac{a}{b}}>1$. Thus from equation (38) we have 


$$
\begin{aligned}
& \frac{1}{\left(s-c-\frac{h+\psi_{1}}{b}\right) e^{b \tau}+s\left(1-r_{2}\right)-\left(\frac{s b-\left(h+\psi_{1}\right)}{b}\right)-\frac{s e^{b \tau}}{a\left(T_{2}-\tau\right)}} \times \\
& {\left[\begin{array}{l}
\left(s-c-\frac{h+\psi_{1}}{b}\right) e^{\alpha_{1} b\left(\tau-t_{1}\right)+b t_{1}}+s\left(1-r_{2}\right)+\left[s\left(1-r_{1}\right) \alpha_{1} b-\left(h+\psi_{1}\right)\right] \\
-e^{\alpha_{1} b\left(\tau-t_{1}\right)}\left(\frac{s b-\left(h+\psi_{1}\right)}{b}\right)-\frac{s e^{\alpha_{1} b\left(\tau-t_{1}\right)}}{a\left(T_{1}-\tau\right)}
\end{array}\right]>1}
\end{aligned}
$$

After simplification we have

$$
\frac{\left(s-c-\frac{h+\psi_{1}}{b}\right) e^{b \tau}}{\left(s-\frac{h+\psi_{1}}{b}\right)-\frac{s\left(1-r_{1}\right) \alpha_{1} b-\left(h+\psi_{1}\right)}{\alpha_{1} b}}>\frac{e^{\alpha_{1} b\left(\tau-t_{1}\right)}-1}{e^{\left(\alpha_{1}-1\right) b\left(\tau-t_{1}\right)}-1}
$$

But $e^{\alpha_{1} b\left(\tau-t_{1}\right)}>e^{\left(\alpha_{1}-1\right) b\left(\tau-t_{1}\right)}$ i.e. $\frac{e^{\alpha_{1} b\left(\tau-t_{1}\right)}-1}{e^{\left(\alpha_{1}-1\right) b\left(\tau-t_{1}\right)}-1}>1$

Therefore, equation (39) yields

$$
\begin{aligned}
& \frac{\left(s-c-\frac{h+\psi_{1}}{b}\right) e^{b \tau}}{\left(s-\frac{h+\psi_{1}}{b}\right)-\frac{s\left(1-r_{1}\right) \alpha_{1} b-\left(h+\psi_{1}\right)}{\alpha_{1} b}}>1 \\
& \Rightarrow\left(s-c-\frac{h+\psi_{1}}{b}\right) e^{b \tau}+\frac{h+\psi_{1}}{b}-s r_{1}>\frac{h+\psi_{1}}{\alpha_{1} b} \Rightarrow r_{1}<\frac{\left(s-c-\frac{h+\psi_{1}}{b}\right) e^{b \tau}}{s}
\end{aligned}
$$

However, by assumption, $r_{1}<1-\frac{c}{s}$.

Therefore, $\tilde{\pi}_{1}>\tilde{\pi}_{2}$ if $r_{1}<\min \left\{1-\frac{c}{s}, \frac{\left(s-c-\frac{h+\psi_{1}}{b}\right) e^{b \tau}}{s}\right\}$ 


\section{Theorem - 2}

$$
\begin{aligned}
& \text { For } n_{1}=n_{2}=n, \tilde{\pi}_{2}>\tilde{\pi}_{3} \text { if } \\
& r_{2}<\min \left\{1-\frac{c}{s}, \frac{s(n-1)+n\left[\left(s b-\left(h+\psi_{1}\right)\right)\left(\frac{e^{b \tau}-1}{b}\right)-\frac{s}{a\left(T_{2}-\tau\right)}-c e^{b \tau}\right]}{s(n-1)}\right\} .
\end{aligned}
$$

\section{Proof:}

The values of $\tilde{\pi}_{2}$ for fixed $r_{2}$ is always less than the optimal value of $r_{2}$. Thus it is sufficient to show that $\tilde{\pi}_{2}>\tilde{\pi}_{3}$ for fixed $r_{2}$. Here $T_{2}$ is the cycle length when post deterioration discount is applied on unit selling price to enhance the demand of decreased quality items. For the enhancement of demand the inventory depletion rate will be higher and consequently the cycle time will reduce $T_{3}$ when no discount is applied on selling price. Obviously $T_{3}$ is greater than $T_{2}$ without loss of generality, let both the profit functions $\tilde{\pi}_{2}$ and $\tilde{\pi}_{3}$ are positive.

$$
\tilde{\pi}_{2}-\tilde{\pi}_{3}=\frac{\tilde{T P}}{T_{2}}-\frac{\tilde{T P}}{T_{3}} \geq \frac{\tilde{T P_{2}}-\tilde{T P}}{T_{3}}
$$

Therefore, it is sufficient to show that $\frac{\tilde{T P_{2}}-\tilde{T P}}{T_{3}}>0$. It can be shown that $\frac{\tilde{T P_{2}}-\tilde{T P}}{T_{3}}>0$ is an increasing function of $r_{2}$ then our purpose will be served. Now, differentiating it with respect to $r_{2}$ we have

$$
\begin{aligned}
\frac{\partial\left(\tilde{\pi}_{2}-\tilde{\pi}_{3}\right)}{\partial r_{2}} & =\frac{1}{T_{3}}\left[\left(s(n-1)\left(1-r_{2}\right)+n \frac{\left(h+\psi_{1}\right)+\theta\left(d+\psi_{2}\right)}{\theta}\right) \frac{a\left(T_{2}-\tau\right)}{\left(1-r_{2}\right)^{n+1}}+\left(s b-\left(h+\psi_{1}\right) \frac{e^{b \tau}-1}{b}\right.\right. \\
& \left.\left.-c e^{b \tau}-\frac{\left(h+\psi_{1}\right)+\theta\left(d+\psi_{2}\right)}{\theta}-\frac{s}{a\left(T_{2}-\tau\right)}\right) \frac{a n\left(e^{\theta\left(T_{2}-\tau\right)}-1\right)}{\theta\left(1-r_{2}\right)^{n+1}}\right]
\end{aligned}
$$

Therefore, $\frac{\tilde{T P}_{2}-\tilde{T P}}{T_{3}}>0$ if $\frac{\partial\left(\tilde{\pi}_{2}-\tilde{\pi}_{3}\right)}{\partial r_{2}}>0$,

i.e., if

$$
\begin{aligned}
& \left(s(n-1)\left(1-r_{2}\right)+n \frac{\left(h+\psi_{1}\right)+\theta\left(d+\psi_{2}\right)}{\theta}\right)+\left[\begin{array}{l}
s b-\left(h+\psi_{1}\right) \frac{e^{b \tau}-1}{b}-c e^{b \tau}- \\
\frac{\left(h+\psi_{1}\right)+\theta\left(d+\psi_{1}\right)}{\theta}-\frac{s}{a\left(T_{2}-\tau\right)}
\end{array}\right] \\
& \frac{n\left(e^{\theta\left(T_{2}-\tau\right)}-1\right)}{\theta\left(T_{2}-\tau\right)}>0
\end{aligned}
$$


Now, $\frac{e^{\theta\left(T_{2}-\tau\right)}-1}{\theta\left(T_{2}-\tau\right)}>1$. Thus from equation (41) we have

$$
s(n-1)\left(1-r_{2}\right)+n\left(\left(s b-\left(h+\psi_{1}\right)\right)\left(\frac{e^{b \tau}-1}{b}\right)-c e^{b \tau}-\frac{s}{a\left(T_{2}-\tau\right)}\right)>0
$$

i.e. $\quad r_{2}<\frac{s(n-1)+n\left(\left(s b-\left(h+\psi_{1}\right)\right)\left(\frac{e^{b \tau}-1}{b}\right)-c e^{b \tau}-\frac{s}{a\left(T_{2}-\tau\right)}\right)}{s(n-1)}$

However, we have the restriction $r_{2}<1-\frac{c}{s}$.

Hence, $\tilde{\pi}_{2}>\tilde{\pi}_{3}$ if

$r_{2}<\min \left\{1-\frac{c}{s}, \frac{s(n-1)+n\left(\left(s b-\left(h+\psi_{1}\right)\right)\left(\frac{e^{b \tau}-1}{b}\right)-c e^{b \tau}-\frac{s}{a\left(T_{2}-\tau\right)}\right)}{s(n-1)}\right\}$

Theorem 1 indicates that for same $n_{1}$ and $n_{2}$ pre and post deterioration discounts on unit selling price produce higher profit than that with only post deterioration discount on unit selling price, if the percentage post deterioration discount on unit selling price is less than $\min \left\{1-\frac{c}{s}, \frac{\left(s-c-\frac{h+\psi_{1}}{b}\right) e^{b \tau}}{s}\right\}$ whereas, Theorem 2 demonstrates that only post deterioration discount on unit selling price is more profitable than profit corresponding to no discount on selling if the percentage post deterioration discount on unit selling price is less than $\min \left\{1-\frac{c}{s}, \frac{s(n-1)+n\left(\left(s b-\left(h+\psi_{1}\right)\right)\left(\frac{e^{b \tau}-1}{b}\right)-c e^{b \tau}-\frac{s}{a\left(T_{2}-\tau\right)}\right)}{s(n-1)}\right\} . \quad$ A simple managerial indication is as follows: in pure inventory scenario if the product deteriorates after a certain time then it is always more profitable to apply both pre and post deterioration discount on unit selling price. The amount of percentage discount must be less than the limit provided in equation (40) for both pre and post deterioration discount. The upper limit for the amount of only post deterioration discount on unit selling price is given in equation (42). 